Superinfection by resistant organisms may follow suppression of the normal bacterial flora in the gastrointestinal tract. Overgrowth with Candida albicans can lead to sore mouth, anorectal inflammation, diarrhoea, and vaginitis. Resistant coliform organisms or staphylococci may cause life-threatening infection in debilitated patients and in those receiving immunosuppressive or corticosteroid therapy.

Renal toxicity-In patients who develop renal insufficiency while taking the drug it may seriously worsen renal function.

Acute benign intracranial hypertension (pseudotumur cerebri) sometimes follows the use of tetracycline in therapeutic doses in infants ; they become irritable, vomit, and develop a tense bulging fontanelle. Adults show meningeal irritation and papilloedema. The spinal fluid is normal and the condition disappears when the drug is stopped.

Hepatotoxicity-Liver damage may follow intravenous use of tetracycline in large doses and has occasionally followed oral use. Such damage (which can be fatal) is more likely to occur in patients with pre-existing hepatic or renal insufficiency, particularly in pregnancy or post partum.

Photosensitivity reactions may occur and manifest as exaggerated sunburn and marked erythema on exposure to sunlight.

Hypersensitivity reactions may take the form of fixed eruptions, urticaria, angioneurotic oedema, non-thrombocytopenic purpura, and rarely anaphylactic shock.

Changes in children's teeth and nails-All tetracyclines are deposited in growing deciduous and permanent teeth, causing yellowish discoloration, enamel hypoplasia and tendency to caries if given to women after the 6th month of pregnancy and to children up to the age of 8 years. They are also deposited in the nails when given to infants and late in pregnancy. The incidence and intensity is related to the total dose of tetracyclines ingested.

\section{Interactions}

Milk, iron, calcium, magnesium, and aluminium salts (present in many antacid preparations) diminish the absorption of tetracycline and therefore none of these should be given at the same time. Oral anticoagulants are potentiated because the drug inhibits vitamin $\mathrm{K}$ synthesising bacteria in the gut. The doses of the oral anticoagulant may thus have to be reduced during tetracycline therapy.
The anaesthetic methoxyflurane has precipitated fatal kidney failure in patients taking tetracycline, and should therefore be avoided.

Dosage required (average dose and dose range)

Oral dose-Uses $(a)$ and $(b)$.

Adults - $1 \mathrm{~g}$ in 2-4 divided doses daily, or $500 \mathrm{mg}$ six-hourly for more serious infections. For long-term prophylaxis in chronic bronchitis $250 \mathrm{mg}$ twice or three times daily.

Children-(see contraindications) $20-40 \mathrm{mg} / \mathrm{kg}$ body weight/day in four divided doses.

(c) Acne vulgaris: $250-500 \mathrm{mg}$ daily as a single dose. The effect may not appear until the drug has been used for two months. Rosacea: $250 \mathrm{mg}$ daily or less usually suffices; some patients need only $250 \mathrm{mg}$ on alternate days or twice weekly.

The eye ointment is applied three or four times daily.

Duration of treatment depends on the use.

\section{PHARMACEUTICAL INFORMATION}

\section{Selected dosage forms}

Capsules contain tetracycline hydrochloride or tetracycline phosphate complex. Usual strengths 250 and $500 \mathrm{mg}$ of tetracycline base.

Oral suspension contains tetracycline hydrochloride or tetracycline phosphate complex. Usual strength $125 \mathrm{mg} / 5 \mathrm{ml}$ (tetracycline base)

Eye ointment-strength $1 \%$.

\section{Storage conditions}

Tetracycline should be stored in an airtight container. The suspension should be protected from light and at a temperature not exceeding $30^{\circ} \mathrm{C}$. Expiry date, two to three years from the date of manufacture, depending on the formulation.

\section{Legal category}

Prescription drug.

\title{
Package inserts for prescribed medicines: what minimum information do patients need?
}

\author{
FREYA HERMANN， A HERXHEIMER， N D W LIONEL
}

British Medical fournal, 1978, 2, 1132-1135

\section{Summary and conclusions}

The information a patient needs about a prescribed medicine can be determined by considering what responsibilities he can assume in relation to taking medicine. When the medicine has been dispensed the patient needs to know how to take the drug; how to store the

School of Pharmacy, Oregon State University, Corvallis, Oregon 97331, USA

FREYA HERMANN, RPH, MS, associate professor of pharmaceutical sciences

Charing Cross Hospital Medical School, London W6 8RF

A HERXHEIMER, MB, FRCP, senior lecturer in clinical pharmacology and therapeutics

Faculty of Medicine, University of Sri Lanka, Colombo 8 N D W LIONEL, MB, FRCP ED, associate professor of pharmacology drug; how it is expected to help; and how to recognise problems and what to do about them. A guide was designed to specify what information is required to meet these needs. Using this guide, a set of minimum information on tetracycline was prepared that aimed at being brief, specific, and readable. The best format for the information remains to be determined.

Since leaflets produced by professional organisations are generally unsuitable for these purposes, information sets should be put together by small independent groups consisting of clinical pharmacologists, clinicians, pharmacists, and consumers. Each country should produce its own sets, adapting model sets to the circumstances of local practice.

\section{Introduction}

If patients are expected to take their medicines, they must be given information. The content, quantity, and format of such information are not easily decided. Patients often demand more (presumably better) information, and indeed, better information may result in better care, though few facts have been presented 
that show the effect, let alone the effectiveness, of giving information to patients. Much investigation and experiment will be needed before we can expect to identify useful ways of providing information about medicines to different groups of patients. Such investigations must concern: the information content; the style and format of presentation; and the circumstances and setting in which the patient receives the information. It is logical to begin by considering information content, and we therefore tried to determine what minimum information a patient needs.

Our approach is based on earlier work done on minimum information for sensible use of self-prescribed medicines, ${ }^{1}$ and on minimum information needed by prescribers. ${ }^{2}$ We began by designing a general guide for constructing information sets for drugs, before assembling information sets for individual drugs. Both the guide and some model sets on individual drugs could be developed by international consensus. We used a draft guide to write information sets for three drugs. The set for tetracycline appears in the appendix; those for propranolol and prescribed aspirin are available from the authors.

\section{The guide}

The guide describes what a patient can do to make his treatment as effective and safe as possible (see table). It lists the types of information that would enable him to do so, and thus provides clear reasons for including specific items in the set of minimum information for an individual drug. The report on minimum information for selfprescribed medicines ${ }^{1}$ suggested that a consensus on minimum information could also be reached for prescribed medicines. But it pointed to the difficulty of finding a universally acceptable demarcation of responsibilities between doctor and patient. Once the patient's responsibilities have been defined, they can be expressed in terms of what he will actually have to do, and from that it follows what information, and how much, he needs.

\section{TO KNOW HOW TO TAKE THE DRUG}

That the patient should know how to take the drug is obvious This means that he must know the dose (1.1) and the manner (1.2) and time (1.3) of taking the dose. It is uncertain how much the patient needs to know about his dose. Often it is enough for him to know, as patients do now from the label, that he should take "two tablets" or "one medicine-spoonful." But sometimes he needs to know the exact dose (1.1.1). He may be asked about the dosage by another doctor whom he has to consult. Secondly, he may be prescribed a variable dose regimen, and then it should be less confusing if instructions were expressed in terms of amount of drug, especially if tablets of different strengths are available. The doctor may also find it easier to instruct a patient whom he expects to change his dose according to circumstances, if that patient knows the amount of drug per dose-for example, a diabetic patient who misses a meal. Average dose and dose range could be useful to the patient $(1.1 .2,1.1 .3)$. Knowing that the dose he is taking is in the usual range may reassure an anxious patient and improve his compliance. How many patients would be helped by knowing more about their dosage, and how many could not use this information, or might be confused by it, requires investigation.

The specific manner in which a dose is to be taken (1.2.1) is often important and even critical. The manner may be dictated by a dosage form, such as enteric-coated tablets which must be swallowed whole (and never taken with milk), or soluble tablets which must be dissolved before taking. It may also be dictated by the drug-for example, tetracycline, which must not be taken with milk, or sulphonamides, which must be taken with large amounts of water. Patients are rarely told the specific time at which the dose should be taken. Yet some drugs are best taken at a particular time of day (1.3.1), such as prednisolone for maintenance treatment (a single morning dose), and those for which the patient needs help in timing his doses, when he has been told only to take the dose "three times daily." If it were ampicillin, he could be told to take it every eight hours. The interval between doses (1.3.2) would also be much clearer for any medicine that is to be taken "as needed." To give the patient the information on the necessary interval between dose and food intake (1.3.3) is critical for medicines whose absorption is decreased by food. The duration of treatment (1.3.4) is important for all those cases when it is necessary to continue taking the drug over a minimum number of days or when it is unwise to continue beyond a maximum number of days.

\section{TO KNOW HOW TO STORE THE DRUG}

Patients must know how to keep their medicines. Storing the medicine is usually simple, but a few must be kept below room temperature, such as antibiotic syrups. Many need to be in tightly closed containers, such as aspirin, or deteriorate faster if they are exposed to light, such as propranolol. If patients store their medicine properly (2.1.1) it is less likely to deteriorate. Of course, medicines bearing an expiry date (2.2.1) should not be kept beyond that date. But a change in the appearance of the medicine (2.2.2) can also warn the patient that it should no longer be used. For example, aspirin tablets that smell of vinegar should be discarded.

Guide to minimum information needed to enable patients to make treatment with a prescribed drug effective and safe

1. To know how to take the drug: 1.1 To take a specific dose

1.2 To take a dose in a specific manner

1.3 To take a dose at specific times

2. To know how to store the drug: 2.1 To store it properly

2.2 To recognise the time at which the medicine becomes subpotent

3. To know how the drug is expected to help:

3.1 To recall the basic facts about the complaint

3.2 To recognise the desired effect and act upon its absence

4. To know how to recognise problems caused by the drug:

4.1 To recognise unwanted effects and act if they occur

4.2 To recall that certain unwanted effects can only be detected by clinical examination or tests

4.3 To recall circumstances indicating need for change of treatment, and act if they occur

4.4 To verify components of medicine

4.5 To act if overdosage occurs
1.1.1 Amount of drug per tablet or other dosage form

$\left\{\begin{array}{l}\text { 1.1.2 } \\ \text { 1.1.3 Average dose and dose range, adult }\end{array}\right.$

1.2.1 Manner directed by dosage form or drug

1.3.1 Clock time

$\left\{\begin{array}{l}\text { 1.3.2 } \\ \text { Time since last dose }\end{array}\right.$

1.3.3 Time since or until food intake

2.1.1 Storage conditions

$\{2.2 .2$ Expiry date

3.1.1 Disease or symptoms to be affected

$\left\{\begin{array}{l}\text { 3.1.2 } \\ \text { 3.1.3 Potential consequence of compliance }\end{array}\right.$

3.2.1 Events showing effect

$\begin{cases}\text { 3.2.2 } & \text { Time at which to expect effect } \\ \text { 3.2.3 Direction for follow-up if there is no effect }\end{cases}$

4.1.1 Events showing unwanted effect

4.1.2 Direction for follow-up of events

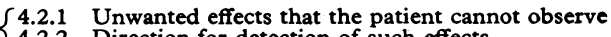

4.2.2 Direction for detection of such effects

4.3.1 Change in the patient's state

4.3.2 Direction for follow-up of chang

4.3.3 Other drug added to therapy

$\left\{\begin{array}{l}\text { 4.4.1 Name of drug and "active" excipien } \\ \text { 4.4.2 Direction for follow-up of "action" }\end{array}\right.$

4.5.1 Direction for follow-up of occurrence 
TO KNOW HOW THE DRUG IS EXPECTED TO HELP

An increasing number of patients want to know how the medicine is expected to help them. Understanding the effect sometimes improves compliance, though it does not necessarily do so. ${ }^{3}$ Arguably, patients should also be able to avoid taking medicine that is unlikely to help them. For this purpose, the patient needs to know the basic facts about his complaint (3.1), how the medicine is likely to affect the disease or symptoms (3.1.1), and what will be the potential consequence of compliance (3.1.2) or non-compliance (3.1.3). He will then know why he should take the medicine. The patient must also know how to recognise the intended effect (3.2.1) and roughly when the effect is to be expected (3.2.2). This is obviously important for drugs with a delayed effect, such as tricyclic antidepressives. The patient must also be told what to do if the therapeutic effect does not occur within a certain time (3.2.3). If the therapeutic effect is one that most patients cannot recognise-for example, a change in blood pressure-the effect needs to be checked by the doctor or nurse. It may help the patient to understand this.

\section{TO KNOW HOW TO RECOGNISE PROBLEMS CAUSED BY THE DRUG}

Many prescribers think that information about unwanted effects may frighten patients and put them off taking a useful medicine. But patients often want such information, and their right to it can hardly be questioned. Unexplained lists of adverse effects can certainly frighten patients and should be avoided. But information given in simple words, that enables the patient to recognise a likely problem (4.1.1), with advice on how to cope with it (4.1.2), may prevent avoidable harm. Patients may not connect new symptoms with the medicine, but a checklist of symptoms may enable them to do so. The patient must also realise that some unwanted effects can be detected only by clinical examination or tests (4.2.1), such as changes in liver function or decrease of blood cells. For these to be detected in time, he must see the doctor regularly (4.2.2.)

In addition, the patient should understand that under certain circumstances a change in treatment may be required. He must be told what they are $(4.3 .1,4.3 .3)$ and what to do $(4.3 .2,4.3 .4)$. For example, a woman who takes an oral anticoagulant should be told that she must tell the doctor if she becomes pregnant. And since a doctor may want to stop propranolol before an operation, or at least would want to know that the patient is taking propranolol, the patient should be instructed to tell any doctor he consults that he is taking propranolol.

Patients must also be told that they need to be careful about taking certain other drugs along with the prescribed medicine. Often this will already have been considered by the doctor and perhaps the pharmacist, but sometimes not, as in the case of a new pregnancy or of self-medication. Appropriate action is then the patient's responsibility. Rarely problems are caused by the so-called "inactive" ingredients, excipients (such as starch or corn syrup), flavouring or colouring agents, or pharmaceutical necessities, such as sodium salts. If patients could verify all components of their medicine (4.4), they could help to protect themselves from an unwanted effect of these substances. They would have to be told the name of the drug and of the inactive ingredients that are known to present problems, the "active" excipients (4.4.1). Advice on what to do if a problem arises is also needed (4.4.2). Patients should also know what to do in case of an accidental overdose (4.5.1).

The guide spells out what the doctor and the pharmacist expect of the patient. Patients are encouraged to take an active part in their treatment and make better use of the doctor and the pharmacist. Experience with the sets of minimum information will show whether patients can use the information and will do so for the purpose for which it is provided. If the design of this guide is shown to be sound, it can be used as a standard for dividing responsibilities between doctor and patient, and between patient and pharmacist. The most immediate use is for writing the sets of minimum information. The task is made relatively easy.

\section{Sets of minimum information about individual drugs}

The guide shows clearly what information and how much should be included in the drug information set and, very important, what can be left out. The information must also be easily understood and convincing. The guide says nothing about the format, but implies that it must be such that the information given will indeed enable the patient to carry out responsibilities, that responsibilities will indeed be assumed, and that this will result in more effective and safer use of the drug. The style and format in which the information is finally presented should aim at brevity, specificity, and readability. Typography, layout, and other aspects of design are important. The cultural and psychological make-up of the patients must be considered. And even before all that, one must decide whether one wants patients to remember the information or to use it only "as needed." In designing experiments that evaluate the information, retention of facts has often been accepted as a measure of success for giving information. Perhaps the process of giving information has been needlessly encumbered by regarding the drug information sheet as a text to be learnt rather than as reference material.

The example for tetracycline (see appendix) shows a format that presents the information for use "as needed." Readability and specificity are most important. The heading introduces the use of the information sheet, and sets the tone of directness and informality. The medicine is fully identified by giving the generic name and the names of the locally available brands. This is meant to reassure the patient, who may know only the brand name, that the information indeed applies to his medicine. The four sections of the information correspond to the four areas of responsibility. This structure underlines the purpose of the information and organises it for later reference. Within each section, information is combined to make it readable, rather than given in the strict sequence of the listing in the guide. For example, all facts telling the patient what the drug does are discussed together. Unwanted effects are grouped together by the action they call for and the urgency of that action, drug interactions are summed up by the direction to ask the pharmacist about all other medicines.

The information was selected from the minimum information for prescribers. $^{2}$ Only information that came within the scope of the guide and could be made useful to the patient was included. It was translated into specific and readable non-technical language. For example, doses are expressed in the same unit of weight as the strength per dosage form. Daily doses are clearly designated as such, to distinguish them from single doses, and intervals between doses are used instead of the number of daily doses. Administration of doses is described in detail. General terms are further interpreted-for example, "on an empty stomach" is changed to "no later than one hour before or no sooner than two hours after meals."

Although patients are commonly warned about problems that may occur with their medicine, they are rarely told what they should do about them. The information sets include specific directions, which clearly state not only whether the patient should seek help, but also how urgently and from whom. For example, in the case of aspirin, the patient would be told to take the tablets with food or milk if he has stomach discomfort, but to stop taking aspirin and to tell the doctor if such discomfort is severe or prolonged. Patients are told to talk to the pharmacist before taking additional medicines and to contact the nearest hospital casualty department in case of accidental overdose. For the overdose, "immediate" action is necessary. For some unwanted effects, such as a pulse slower than 55 beats per minute while on propranolol, the doctor should be "told promptly," but if minor side effects occur, such as nausea or sleeplessness, the doctor need only be "informed."

In summary, style and format were chosen to support the intent of the guide. Their usefulness needs to be validated when the effectiveness of the information sets is assessed.

\section{Relation to other information for patients}

The minimum information package is intended for the patient who has been given a specific medicine. Nevertheless, the patient may 0 need other information at other times. For example, just before the $\mathbb{D}$ doctor considers prescribing at all, doctor and patient both need to $\stackrel{\mathscr{P}}{?}$ consider the likely benefits, contraindications and risks, inconveniences, 0 and possible costs of the various choices. Ideally, the doctor will inform and guide the patient on these matters within the limits of $\overrightarrow{\mathbb{D}}$ the patient's ability. The doctor has been shown to be the person $\frac{?}{\mathbb{1}}$ from whom patients are most likely to accept information on medi- $\varrho$ cines, and for practical purposes it is best given at the time of prescribing. If the patient gets the information later he has been denied 8 his chance to use it, since he has already accepted the treatment. Rarely will a patient be so determined that he will reject the medicine that has already been dispensed, return to the doctor and ask for

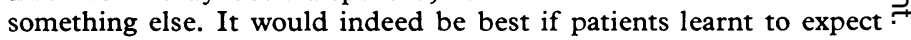
this information during the consultation and asked the doctor about points that they have not fully understood. A list of questions about medicines that patients may wish to ask the doctor has been suggested. ${ }^{4}$ 
If patients were to get used to considering benefits, risks, and inconveniences with their doctor, they would probably consider the same points when choosing self-prescribed (over-the-counter) medicines. ${ }^{1}$ In the pharmacy the patient may also need more information. Pharmacists are in an excellent position to know what medicines the patient is taking, since they may dispense all the prescriptions a patient receives and know what self-prescribed medicines he uses. Pharmacists can protect patients from harmful interactions. Ideally, the pharmacist will also help with information on cost and equivalence of alternative preparations, especially when patients pay for the medicine and wish to spend as little as possible.

\section{Who should be responsible for providing sets of minimum information for patients?}

Patients have seldom been given information on individual medicines. A few package inserts for patients exist. In countries where inserts originally intended for doctors are given to patients, manufacturers have amended them to make them more useful to patients. But manufacturers have to work under constraints which make it difficult to produce sets of minimum information such as we have proposed. For example, it may be illegal to refer to existing medical uses that have not yet been accepted by the regulatory agency. On the other hand, manufacturers have been obliged to list every adverse effect, and listings have become excessively comprehensive. Professional organisations have also designed information leaflets, but these have generally not covered individual drugs, nor have they been generally available for distribution.

We therefore believe that, as with the minimum information for prescribers, ${ }^{2}$ sets of minimum information for patients should be put together by a small independent group, consisting of clinical pharmacologists, clinicians, pharmacists, and consumers. Since the extent of the information needed will vary from country to country, each country should produce its own sets of minimum information for patients, using model sets as a standard.

We thank the many colleagues and manufacturers who commented on drafts of this paper and on the sets of minimum information. This work was supported by the International Organisation of Consumer Unions (IOCU) and Consumers' Association (UK).

Requests for reprints should be sent to Dr A Herxheimer.

\section{References}

${ }^{1}$ Ad-hoc Working Group, Lancet, 1977, 2, 1017.

2 Herxheimer, A, and Lionel, N D W, British Medical fournal, 1978, 2, 1129.

${ }^{3}$ Sackett, D L, et al, Lancet, 1975, 1, 1205.

${ }^{4}$ Herxheimer, A, Lancet, 1976, 2, 1294.

(Accepted 18 August 1978)

\section{Appendix}

MINIMUM INFORMATION CONTAINED IN LEAFLET ON TETRACYCLINE

Please read this leaflet carefully before you start taking the medicine. If you have questions, ask your doctor (or dentist) or pharmacist.

\section{What you should know about treatment with}

Tetracycline Brand name: A, B, C

WHAT THE MEDICINE DOES

(a) It is an anti-infective medicine which acts against a wide range of germs by disturbing important life processes within them. In the patient the effect is decrease of all signs of the disease, usually beginning within two days. If the expected effect does not occur tell your doctor promptly.
The germs are still present even after the signs of disease have decreased greatly and the need for treatment continues. The medicine must be taken as prescribed by your doctor until the course of treatment is finished, otherwise the infection is likely to flare up again.

(b) In acne the medicine is very effective but the effect may not be noticeable until the drug has been used for two months.

\section{TAKING THE MEDICINE, IMPORTANT DETAILS}

Take only as directed by your doctor (or dentist)

Use only for the patient for whom it was prescribed.

Capsules contain: $250,500 \mathrm{mg}$ tetracycline.*

Oral suspension contains: $125 \mathrm{mg}$ tetracycline in each $5 \mathrm{ml} .^{*}$ Adults

Acute infections:

Usual dose: $250 \mathrm{mg}$.

Usual dose range: $250-500 \mathrm{mg}$.

The dose is taken every six hours. 500 -mg doses may be taken every six hours or only every 12 hours.

Acne and rosacea:

Usual dose: $250 \mathrm{mg}$ daily.

In rosacea, twice weekly doses may be enough.

Usual dose range: $250-500 \mathrm{mg}$ daily.

The dose is taken as a single dose every 24 hours.

Maintenance treatment in chronic bronchitis:

Usual dose: $250 \mathrm{mg}$.

Children

The dose is taken every eight or 12 hours.

The medicine is not recommended for children under 8 years because it interferes with bone growth and may permanently stain developing teeth.

8-12 years

Usual dose depends on the child's weight.

Usual dose range: $20-40 \mathrm{mg}$ per $\mathrm{kg}$ of weight, daily.

The daily dose is taken, divided into four equal doses, one dose every six hours.

The medicine is most effective if it is taken on an empty stomach.

Take it no later than one hour before meals and no sooner than two hours after meals.

Take it at regular intervals, so far as possible every six hours, at the exact time for which it was prescribed.

Capsules: swallow whole with water.

Suspension: shake well before measuring the dose and take with water.

Do not take this medicine with milk or any drink containing milk.

STORING THE MEDICINE

Store the medicine in a tightly closed container, protected from light and from temperatures above $30^{\circ} \mathrm{C}$

Discard the medicine after the expiry date.

Keep the medicine out of reach of children.

\section{POSSIBLE PROBLEMS}

Tetracycline is much less effective if it is taken with milk-so take it with water.

Tetracycline may make you very sensitive to sunlight-so be cautious about sunbathing.

Tetracycline complicates the use of other medicines such as anticoagulant tablets, preparations containing iron, or any preparation for indigestion containing calcium, magnesium, or aluminium-if you see any doctor other than your own, tell him that you are taking tetracycline; before you use any medicine other than this, ask your pharmacist.

If problems occur do not try to treat them with a home remedy; do not stop the medicine on your own.

Should you have severe or prolonged nausea or heartburn, wind, diarrhoea, sore mouth, or anal irritation-inform your doctor.

Should you have skin rash-tell your doctor promptly.

During long-term treatment various body functions may change, and treatment may have to be changed-you therefore need to see your doctor regularly.

Tetracycline should not be used in pregnancy and while breastfeeding - so tell your doctor if you become pregnant.

*Important inactive ingredients should be listed here. 